\title{
(C) OPEN ACCESS \\ Increased risk of non-multiple sclerosis demyelinating syndromes in patients with preexisting septicaemia: a nationwide retrospective cohort study
}

\author{
Chung-Hsing Chou, ${ }^{\oplus 1,2}$ Jiunn-Tay Lee, ${ }^{1,2}$ Chia-Kuang Tsai, ${ }^{1,2}$ Li-Ming Lien, ${ }^{3,4}$ \\ Jiu-Haw Yin ${ }^{1,5}$ Chun-Chieh Lin, ${ }^{1}$ I-Ju Tsai, ${ }^{6}$ Yueh-Feng Sung, ${ }^{1}$ Fu-Chi Yang, \\ Chia-Lin Tsai, ${ }^{1}$ I-Kuan Wang, ${ }^{7,8,9}$ Chun-Hung Tseng, ${ }^{10}$ Chung-Y Hsu ${ }^{7}$
}

\begin{abstract}
- Additional material is published online only. To view please visit the journal online (http://dx.doi.org/10. 1136postgradmedj-2019136667).
\end{abstract}

For numbered affiliations see end of article.

\section{Correspondence to} Jiunn-Tay Lee, Department of Neurology, Tri-Service General Hospital, National Defense Medical Center, Taipei, Taipei, Taiwan, Republic of China; jiunntay@gmail.com

Received 17 April 2019 Revised 15 May 2019 Accepted 30 May 2019 Published Online First 17 June 2019

Check for updates

(c) Author(s) (or their employer(s)) 2019. Re-use permitted under CC BY-NC. No commercial re-use. See rights and permissions. Published by BMJ.

To cite: Chou C-H, Lee J-T,

Tsai C-K, et al.

Postgrad Med J

2019:95:307-313.

\begin{abstract}
Background Growing evidence shows links between septicaemia and non-multiple sclerosis demyelinating syndromes (NMSDS); nevertheless, epidemiological data are still very limited. This study aimed to explore the relationship between septicaemia and NMSDS in a general population.

Methods The study included 482781 individuals diagnosed with septicaemia and 1892825 age/sexmatched non-septicaemia patients for the comparison. Data were drawn from a population-based nationwide National Health Insurance Research Database Taiwan, from 1 January 2002 to 31 December 2011. The two cohorts of patients with and without septicaemia were followed up for the occurrence of NMSDS. The Coxproportional hazard regression model was performed to estimate adjusted HR after multivariate adjustment.

Results Individuals with septicaemia had a 4.17-fold (95\% Cl 3.21 to 5.4, p < 0.001) higher risk to develop NMSDS compared with those without septicaemia. Patients aged $<65$ years had a greater NMSDS risk $(<45$ years: $H R=6.41,95 \% \mathrm{Cl} 3.65$ to $11.3, p<0.001 ; 45-$ 64 years: $H R=6.66,95 \% \mathrm{Cl} 3.98$ to $11.2, p<0.001)$. Furthermore, females with septicaemia and individuals with higher severity of septicaemia were associated with increased risks of developing NMSDS.

Conclusions Our results indicated that patients with septicaemia were likely to develop NMSDS. A possible contributing role of septicaemia in increasing the hazard of NMSDS is proposed, based on the outcome that individuals with higher severity of septicaemia carried elevated threat of encountering NMSDS.
\end{abstract}

\section{INTRODUCTION}

Oligodendrocytes are responsible for myelin production in the central nervous system (CNS). ${ }^{1}$ The myelin sheaths cover and insulate axons which assist electrical conduction between nerves. Demyelinating disorders following oligodendrocyte injuries denote the loss of the myelin sheath that encloses and defends axons in the CNS. Demyelination is likely to be patchy and segmented, with diverse distinctive areas being involved chronologically or concurrently. Individuals with demyelinating diseases present with various symptoms and signs, such as blurred vision, muscle weakness, poor balance and neurocognitive impairment. ${ }^{2}$ The neural function might recover gradually through regeneration and repair of myelin as remyelination does occur. Nevertheless, widespread loss of myelin commonly results in irremediable degeneration of the axon and the cell body. A comprehensive investigation on risk factors of CNS demyelinating diseases might provide better solutions to disease prevention.

Sepsis is a globally common, lethal and costly disease. Based on the third international consensus, the current definition of sepsis is life-endangering organ dysfunction triggered by a poorly controlled host immune response to infection. ${ }^{3}$ Septic shock, a subset of sepsis in which predominantly overwhelming circulatory, cellular and metabolic malformations, has increased hazard of mortality compared with sepsis alone. ${ }^{3}$ Septicaemia is sepsis in the presence of multiplication of pathogenic microorganisms in the circulating blood. Several cases with CNS demyelinating diseases, such as transverse myelitis and acute disseminated encephalomyelitis (ADEM), were reported to be associated with septicaemia. ${ }^{45}$ However, the association between septicaemia and demyelinating diseases remains to be elucidated, with a lack of an appropriate longitudinal follow-up survey.

In this study, we focused on non-multiple sclerosis demyelinating syndromes (NMSDS), which mainly comprised neuromyelitis optica spectrum disorders (NMOSD) and antimyelin oligodendrocyte glycoproteinantibody (MOG) associated spectrum, after septicaemia. We excluded multiple sclerosis (MS) because previous studies demonstrated that MS is a complex disease. ${ }^{6}$ Genetic susceptibility $^{7}$ and various environmental factors, such as vitamin D deficiency, ${ }^{8}$ parental smoking ${ }^{9}$ and adverse socioeconomic position, ${ }^{10}$ have been proposed to be connected to the pathophysiology of MS. Therefore, we aimed to explore the association between septicaemia and NMSDS using the nationwide 10-year follow-up data (2002-2011) in Taiwan. We then theorised that individuals with septicaemia had a greater risk of developing subsequent NMSDS.

\section{METHODS}

\section{Subject enrolment}

To evaluate the association between septicaemia and the subsequent NMSDS, Longitudinal Health Insurance Database (LHID) Taiwan was applied to this study. It is a subdata set of National Health 
Insurance (NHI) programme which contains over 99\% of Taiwanese citizens (23 million). The LHID from 2002 to 2011 encloses the records of sociodemographic status, dates of clinical visits, medications and disease diagnosis codes of inpatients and outpatients in the format of the International Classification of Disease, Ninth Revision, Clinical Modification (ICD-9-CM). All data have been deidentified before available for research. The data of all patients with an index date, that is, the first hospitalisation due to septicaemia, between 2002 and 2011 were followed up and completed until the end of 2014. All protocols in this study were approved by Institutional Review Board of China Medical University Hospital (CMUH104-REC2-115(CR-2)).

\section{Patient and public involvement}

Patients and public were not directly involved in the development of this study.

\section{Definition of septicaemia and demyelinating disease by ICD classification}

The study population included all individuals who had first time hospitalisation for septicaemia with specific ICD-9-CM codes 003.1, 036.2 and 038 from 2002 to $2011(\mathrm{~N}=482781)$. The definition of 'first time' is that those patients were never hospitalised for septicaemia before by searching throughout the National Health Insurance Research Database (NHIRD) Taiwan. The Inpatient Expenditures by Admissions was from 1996 to 2013. In this study, we selected patients with newly diagnosed septicaemia so that we could know the complete history of the patients after having septicaemia. NHIRD is a database maintained by the government, and the missing rate is very low. The data we used in the present study were all completed.

We excluded patients with a diagnosis of MS (ICD-9-CM code 340) and those with other demyelinating diseases (ICD-9-CM code 341) before the index date. The NMSDS here mainly comprise NMO and NMOSD, which are mediated by aquaporin-4 antibodies, as well as anti-MOG associated spectrum, which often presents as an anti-MOG autoimmune encephalomyelitis, chronic relapsing inflammatory optic neuritis, ADEM or acute haemorrhagic leukoencephalitis. Some patients may be classified as unspecified demyelinating diseases of CNS, instead of currently identified specific demyelinating diseases. We arbitrarily recruited an age/sex-matched control group, 1892825 individuals (a sample size 4-fold that of the septicaemia group), from the LHID (2002-2011). Each subject in both groups was followed up from the index date to the event of being diagnosed as demyelinating diseases. For individuals who did not develop demyelinating diseases, the follow-up ended on the date of insurance withdrawal, death or the final day of the research period (31 December 2014) (figure 1).

The associated comorbidities were selected as the following ICD codes: systemic lupus erythematosus (ICD-9-CM code 710), depression (ICD-9-CM codes 296.2, 296.3, 300.4, 301.12, 309.0, 309.1, 311), peripheral vascular disorders (ICD-9-CM codes 440, 441.2, 441.4, 441.7, 441.9, 443, 444, 447.1), deficiency anaemias (ICD-9-CM codes 280 and 281), rheumatoid arthritis (ICD-9-CM code 714), fluid and electrolyte disorders (ICD-9-CM code 276), tobacco use disorders (ICD-9-CM codes 305.1 and 649.0) and infectious mononucleosis (ICD-9-CM code 075).

\section{Statistical analyses}

SPSS V.22.0 was used for the statistical analyses. Group differences in continuous variables were investigated using independent samples t-tests. Pearson's $\chi^{2}$ tests were used to compare the distribution of categorical variables between the septicaemia and non-septicaemia groups. Furthermore, the multivariate Cox proportional hazards model was applied to estimate the risk factors of NMSDS, and the outcome was shown as HRs with 95\% CIs. To examine the interaction between covariates associated with septicaemia and NMSDS, we computed adjusted HRs, stratified by length of hospitalisation due to septicaemia (1-5 days or more than 5 days) and severity of septicaemia. Age ( $<45$ years, $45-64$ years and $\geq 65$ years), sex and comorbidities were included in a regression model. All tests were two-sided, and significant differences were indicated when the two-sided $\mathrm{p}$ values were less than 0.05 .

\section{RESULTS}

\section{Demographics of the study population}

There were 482781 patients with a diagnosis of septicaemia and 1892825 non-septicaemia controls recruited in this retrospective cohort study. The demographic characteristics were shown in table 1 . The mean age was about 59 years old and most individuals in both cohorts were male $(52.1 \%$ and $52.4 \%$ in septicaemia and non-septicaemia groups, respectively). The age distribution differed from $0.1 \%$ to $1 \%$ in each group and the mean age difference was only 0.7 years, which was very similar between the two groups. However, the population in this study was very large, so even the age distribution was very close between the two groups, the p-value was still significant. There was a higher prevalence of all the comorbidities including systemic lupus erythematosus, depression, peripheral vascular disorders, deficiency anaemias, rheumatoid arthritis, fluid and electrolyte disorders, smoking, infectious mononucleosis in the septicaemia group.

The result showed that 144 septicaemia patients developed subsequent NMSDS with an overall rate of 6.91 cases per 100 000 person-years. There were 136 patients having NMSDS in the comparison group with an overall rate of 1.10 cases per 100000 person-years. The data disclosed that patients with septicaemia had 4.17 times $(95 \%$ CI 3.21 to $5.4, \mathrm{p}<0.001)$ higher risk of acquiring NMSDS compared with those without septicaemia (table 2). A list of different diagnoses of NMSDS is provided in online supplementary table 1 .

We further investigated the impact of severity of septicaemia on the risk of developing NMSDS. First, individuals hospitalised due to septicaemia for 1-5 days or more than 5 days have increased risks of developing NMSDS, with HRs 2.17 (95\% CI 1.29 to $3.69, \mathrm{p}<0.001)$ and $4.73(95 \%$ CI 3.62 to $6.18, \mathrm{p}<0.01)$, respectively. Second, patients who had been hospitalised due to septicaemia for more days during the follow-up period had an elevated risk of developing NMSDS (severe group: $\mathrm{HR}=18.7$, $95 \%$ CI 13.7 to $25.6, \mathrm{p}<0.001$ and moderate group: $\mathrm{HR}=3.35$, $95 \%$ CI 2.25 to $4.98, \mathrm{p}<0.001)$. The risk of septicaemia-related NMSDS declined in the group with mild severity (the first tertile) and showed no significant difference compared with the matched controls ( $\mathrm{HR}=0.83$, 95\% CI 0.47 to $1.45, \mathrm{p}>0.05)$. In tables 2 and 3, Cox regression models were conducted. One was for all speticaemia patients versus comparison group and the comparison group was the reference group. Another was the length of stay, 1-5 days, $>5$ days versus the comparison group and the comparison group was the reference group. The other was the severity of speticaemia, mild, moderate, severe versus the comparison group and the comparison group was the reference group. 


\section{3 million NHI beneficiaries from NHIRD}

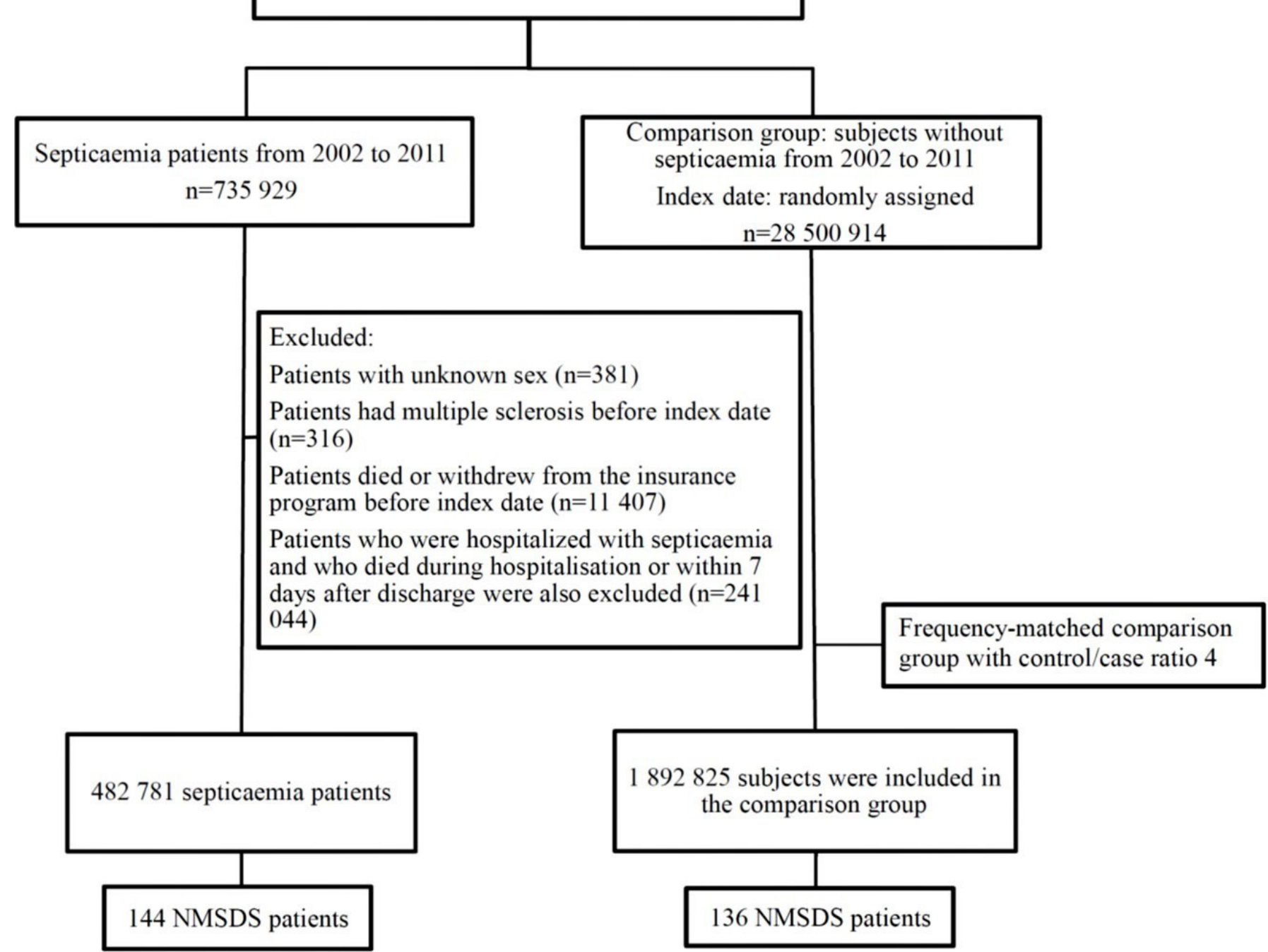

Figure 1 Flowchart represents the steps of sample selection from National Health Insurance Research Database (NHIRD) Taiwan. NHI, National Health Insurance; NMSDS, non-multiple sclerosis demyelinating syndromes.

The results of NMSDS risks after adjusting age $(<45,45-64$ and $\geq 65$ years), sex and comorbidities are listed in table 3 . One regression model for each stratification, and the reference group was the comparison group. Totally, seven Cox regression models were conducted. In tables 2 and 3, all Cox regression models were adjusted for the same covariates which include age, sex and comorbidities listed in table 1. The HR of developing NMSDS was significantly elevated in the septicaemia group, in patient groups, including $<45$ years $(\mathrm{HR}=6.41,95 \% \mathrm{CI} 3.65$ to 11.3 , $\mathrm{p}<0.001)$ and $45-64$ years $(\mathrm{HR}=6.66,95 \%$ CI 3.98 to 11.2 , $\mathrm{p}<0.001)$. The impact of septicaemia on NMSDS was relatively mild in the group $\geq 65$ years $(\mathrm{HR}=2.45,95 \%$ CI 1.65 to 3.62 , $\mathrm{p}<0.001)$. We also investigated whether septicaemia is a sex-dependent risk factor for acquiring NMSDS. The Cox regression analysis demonstrated that the increased risk of NMSDS in septicaemia females ( $\mathrm{HR}=4.43,95 \%$ CI 3.12 to 6.29 , p < 0.001) was higher than septicaemia males $(\mathrm{HR}=3.89,95 \%$ CI 2.63 to 5.75 , $\mathrm{p}<0.001)$.

The influence of comorbidities on the risk of developing NMSDS was also investigated. Interestingly, the septicaemia patients with and without comorbidities had similar HR of developing NMSDS compared with the respective control groups $(\mathrm{HR}=3.74,95 \% \mathrm{CI} 1.50$ to $9.27, \mathrm{p}<0.01$ and $\mathrm{HR}=3.95$, $95 \%$ CI 3.01 to $5.2, \mathrm{p}<0.001$, respectively). Details of chronic comorbidities including other immunocompromise or autoimmune disease in case and comparison groups are provided in online supplementary table 2. Moreover, the Kaplan-Meier analysis demonstrated that patients with septicaemia had significantly higher incidence of NMSDS compared with the matched controls (log-rank test $\mathrm{p}<0.0001$, figure 2 ).

\section{DISCUSSION}

Postinfectious demyelinating diseases, including myelitis, ADEM and acute transverse myelitis have been reported. ${ }^{41112}$ However, most of previously reported cases are children and only a few adults. By studying a representative database of Taiwanese population, this 10-year longitudinal study investigated the association between septicaemia and the risk of developing NMSDS. The result suggests that septicaemia patients had an increased risk of developing NMSDS, especially in females, age $<65$ years and those with higher severities of septicaemia. 
Table 1 Baseline characteristics

\begin{tabular}{|c|c|c|c|}
\hline & Septicaemia & Comparison group & \multirow[b]{2}{*}{$P$ value } \\
\hline & $(n=482781)$ & $(n=1892825)$ & \\
\hline \multicolumn{4}{|l|}{ Age, years } \\
\hline$<18$ & $44198(9.2)$ & $176792(9.3)$ & $<0.0001$ \\
\hline $18-64$ & $185350(38.4)$ & $741400(39.2)$ & \\
\hline$\geq 65$ & $253233(52.5)$ & $974633(51.5)$ & \\
\hline Mean (SD) & $59(24.7)$ & $58.3(24.5)$ & \\
\hline \multicolumn{4}{|l|}{ Sex, $n(\%)$} \\
\hline Female & 231254 (47.9) & $901921(47.6)$ & 0.0018 \\
\hline Male & $251527(52.1)$ & $990904(52.4)$ & \\
\hline \multicolumn{4}{|l|}{ Comorbidity, n (\%) } \\
\hline Systemic lupus erythematosus & $2660(0.6)$ & $1409(0.1)$ & $<0.0001$ \\
\hline Depression & 17834 (3.7) & $22843(1.2)$ & $<0.0001$ \\
\hline Peripheral vascular disorders & $24252(5.0)$ & $31928(1.7)$ & $<0.0001$ \\
\hline Deficiency anaemias & $53278(11.0)$ & $62885(3.3)$ & $<0.0001$ \\
\hline Rheumatoid arthritis & $5301(1.1)$ & $7354(0.4)$ & $<0.0001$ \\
\hline Fluid and electrolyte disorders & $181843(37.7)$ & $191102(10.1)$ & $<0.0001$ \\
\hline Smoking & $61(0.0)$ & $110(0.0)$ & $<0.0001$ \\
\hline Infectious mononucleosis & $734(0.2)$ & $657(0.0)$ & $<0.0001$ \\
\hline
\end{tabular}

Septicaemia is a severe systemic infection that is associated with life-menacing organ dysfunction triggered by unbalanced host immune response. In the present study, we focused on the association between the development of NMSDS and preexisting septicaemia, which was defined as a serious infection which spreads through the entire vascular system of the body. Septicaemia could impair CNS function via a variety of mechanisms, including metabolic dysfunction, ${ }^{13}$ oxidative stress ${ }^{14}$ and microcirculatory impairment, ${ }^{15}$ all of which could instigate sustained damage to neurovascular structure. Currently, septicaemia has been found to be a risk factor for cognitive impairment, ${ }^{16}$ dementia $^{17}$ and stroke. ${ }^{18}$ Our data revealed that septicaemia might have a lasting influence on increasing the risk for NMSDS.
There was a significant higher risk of developing NMSDS in the relatively young age group ( $<65$ years) compared with the elderly ( $\geq 65$ years) in our study. The NMSDS associated with several autoimmune disorders, such as giant cell arteritis rather than preexisting septicaemia, are relatively common in the elderly. ${ }^{19}$ In our study, the portion of patients with giant cell arteritis was very low (less than $0.1 \%$ in both cohorts, online supplementary table 2). In contrast, fibromyalgia was relatively common in group age $45-64(1.6 \%$ and $0.6 \%$ in speticaemia and comparison groups respectively, online supplementary table 2). The finding is in agreement with a previous study. ${ }^{20}$ In our study population, the prevalence of comorbidity such as fibromyalgia was higher than giant cell arteritis, and these middle age septicaemia patients with fibromyalgia are relatively prone to have demyelinating syndromes, including MS. ${ }^{21}$ This may explain in part the observation that the 45-64 age group with pre-exsiting septicaemia had the highest adjusted HR of developing NMSDS. Age-related variance of immune response was therefore suggested to be associated with development of subsequent demyelination after systemic infection. We also found that females with septicaemia had a higher risk of developing NMSDS compared with male patients.

Systemic infections may induce damage to the CNS even though there is no evidence of direct invasion. Mawanda et al reported that extra-CNS bacterial infection can trigger diverse cognitive dysfunction with an increased risk for dementia. ${ }^{22}$ Lindsberg and Grau reported that acute and exacerbating chronic infection may contribute to consequent strokes. $^{23}$ Various mechanisms have been proposed to explain the impact of systemic infections to CNS inflammation, which may further induce demyelination disease due to oligodendrocyte injuries.

First, the connections between neurons rely on electrical and chemical signals which are controlled within a narrow and stable homeostatic regulation. The integration of blood brain barrier $(\mathrm{BBB})$ is critical for maintenance of a precise extracellular milieu around synapses and axons. BBB was mainly composed of astroglial foot processes, endothelial cells and pericytes. ${ }^{24}$ Dysfunction of BBB resulting from the endothelial

Table 2 Incidence of subsequent demyelinating diseases of the central nervous system, rather than multiple sclerosis, stratified by severity of septicaemia

\begin{tabular}{|c|c|c|c|c|c|c|}
\hline & $\mathrm{N}$ & Event & PYs & Rate & Crude HR $(95 \% \mathrm{CI})$ & Adjusted HR $(95 \% \mathrm{Cl})$ \\
\hline \multicolumn{7}{|l|}{ All } \\
\hline Comparison group & 1892825 & 136 & 12352780 & 1.10 & Reference group & Reference group \\
\hline Septicaemia & 482781 & 144 & 2082900 & 6.91 & $5.99(4.73 \text { to } 7.58)^{* * *}$ & $4.17(3.21 \text { to } 5.4)^{* * *}$ \\
\hline \multicolumn{7}{|c|}{ Length of stay due to septicaemia* } \\
\hline Comparisons & 1892825 & 136 & 12352780 & 1.10 & Reference group & Reference group \\
\hline $1-5$ days & 91289 & 16 & 523515 & 3.06 & $2.73(1.63 \text { to } 4.59)^{* * *}$ & $2.17(1.27 \text { to } 3.69)^{* * *}$ \\
\hline$>5$ days & 391492 & 128 & 1559385 & 8.21 & $7.06(5.54 \text { to } 9.01)^{* * *}$ & $4.73(3.62 \text { to } 6.18)^{* *}$ \\
\hline$P$ for trend & & & & & $<0.0001$ & $<0.0001$ \\
\hline \multicolumn{7}{|l|}{ Severity of septicaemia } \\
\hline Comparisons & 1892825 & 136 & 12352780 & 1.10 & Reference group & Reference group \\
\hline Mild (T1) & 144548 & 14 & 1115282 & 1.26 & 1.12 (0.64 to 1.93$)$ & 0.83 (0.47 to 1.45$)$ \\
\hline Moderate (T2) & 145160 & 34 & 657778 & 5.17 & $4.92(3.36 \text { to } 7.19)^{* * *}$ & $3.35(2.25 \text { to } 4.98)^{* * *}$ \\
\hline Severe (T3) & 193073 & 96 & 309840 & 31.0 & $28.5(21.4 \text { to } 38.0)^{* * *}$ & $18.7(13.7 \text { to } 25.6)^{* * *}$ \\
\hline$P$ for trend & & & & & $<0.0001$ & $<0.0001$ \\
\hline
\end{tabular}

Models adjusted for age, sex and comorbidities listed in table 1. Severity = (total length of hospital stay due to septicaemia during the follow-up duration) $\div$ (length of follow-up duration). $\mathrm{T} 1$, the first tertile: $<0.44 \%$; $\mathrm{T} 2$, the second tertile: $0.44 \%-1.58 \%$; $\mathrm{T} 3$ the third tertile: $>1.58 \%$.

${ }^{* *} p<0.01 ;{ }^{* * *} p<0.001$.

*The first time hospitalisation due to septicaemia.

PYs, person-years; Rate, incidence rate, per 100000 person-years. 
Table 3 Risk of demyelinating diseases of the central nervous system, rather than multiple sclerosis, in patients with and without septicaemia, stratified by age, sex and comorbidity

\begin{tabular}{|c|c|c|c|c|c|c|c|}
\hline \multirow[t]{2}{*}{ Variable } & \multicolumn{3}{|c|}{ Septicaemia cohort } & \multicolumn{3}{|c|}{ Comparison cohort } & \multirow[t]{2}{*}{ Adjusted HR $(95 \% \mathrm{Cl})$} \\
\hline & Event & PYs & Rate & Event & PYs & Rate & \\
\hline \multicolumn{8}{|l|}{ Age group } \\
\hline$<45$ & 47 & 753379 & 6.24 & 21 & 3489202 & 0.60 & $6.41(3.65 \text { to } 11.3)^{* *}$ \\
\hline $45-64$ & 51 & 531048 & 9.6 & 29 & 3283699 & 0.88 & $6.66(3.98 \text { to } 11.2)^{* *}$ \\
\hline$\geqq 65$ & 46 & 798474 & 5.76 & 86 & 5579879 & 1.54 & $2.45(1.65 \text { to } 3.62)^{* *}$ \\
\hline \multicolumn{8}{|l|}{ Sex } \\
\hline Female & 87 & 1060254 & 8.21 & 68 & 5924056 & 1.15 & $4.43(3.12 \text { to } 6.29)^{* *}$ \\
\hline Male & 57 & 1022646 & 5.57 & 68 & 6428724 & 1.06 & $3.89(2.63 \text { to } 5.75)^{* *}$ \\
\hline \multicolumn{8}{|c|}{ Comorbidities } \\
\hline No & 115 & 1893501 & 6.07 & 130 & 12135849 & 1.07 & $3.95(3.01 \text { to } 5.2)^{* *}$ \\
\hline Yes & 29 & 189399 & 15.31 & 6 & 216931 & 2.77 & $3.74(1.50 \text { to } 9.27)^{*}$ \\
\hline
\end{tabular}

Models adjusted for age, sex and comorbidities listed in table 1.

${ }^{* *} p<0.01 ;{ }^{* *} p<0.001$.

PYs, person-years; Rate, incidence rate, per 100000 person-years.

nitric oxide synthase (e-NOS) related oxidant stress in early stage of systemic inflammation has been demonstrated in animal studies. ${ }^{25}$ The e-NOS was found to be associated with disturbed cerebral autoregulation in sepsis due to its proinflammatory properties, which contribute to malfunction of cerebrovascular endothelial cells. ${ }^{25} 26$ Impaired cerebral autoregulation contributes to cerebral hypoperfusion and further ischaemic damage to brain tissues in those with septic shock. ${ }^{2627}$ Moreover, lipopolysaccharides could induce oligodendrocyte damage via activation of neuronal NOS in oligodendrocytes and then cause demyelination. ${ }^{28}$

Second, severe infections cause systemic inflammation with significantly elevated concentrations of inflammatory cytokines, such as tumour necrosis factor- $\alpha$, interleukin $1 \beta$ (IL-1 $\beta$ ) and IL-6. ${ }^{29}{ }^{30}$ Animal studies provided evidence that the circulating cytokines could activate the microglia/macrophages in the CNS, which further induced neuronal damage by secreting inflammatory mediators. ${ }^{31} 32$ Furthermore, oligodendrocyte injuries evidenced by development of demyelination lesions after direct injection of inflammatory cytokines have been demonstrated in transgenic mice with overexpression of IL-6 and interferon-gamma. ${ }^{33}$ All of these studies supported the hypothesis that cytokines mediate demyelination. Additionally, disruption of

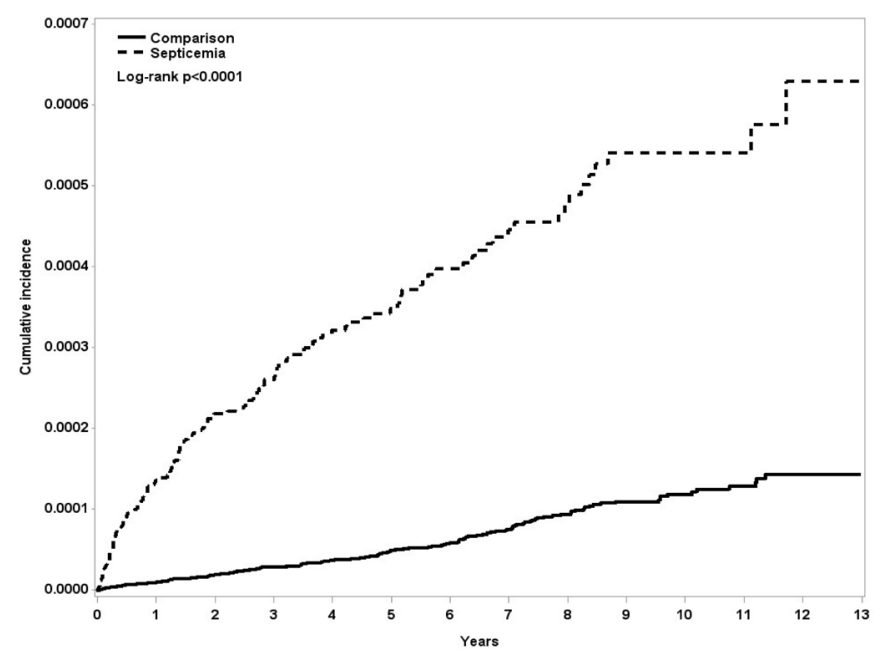

Figure 2 The cumulative incidence curves of non-multiple sclerosis demyelinating syndromes for individuals with and without septicaemia.
BBB allows more inflammatory cytokines crossing into the CNS, which has been proved by the findings of higher concentrations of CSF proteins in septic patients compared with non-septic controls. $^{35}$

Third, molecular mimicry plays an important role in autoimmunity. Many experiments, using peptides from viral or bacterial pathogen, have successfully activated the autoreactive $\mathrm{T}$ cells and caused damage to normal tissues. ${ }^{36} 37$ Therefore, $\mathrm{T}$ cell-mediated autoimmunity could be triggered by microbial peptides that have proper structural resemblance to self-peptides. There are case reports of CNS demyelinating diseases after infection with viral or bacterial pathogens, such as Salmonella bacteremia, ${ }^{12}$ Epstein-Barr virus ${ }^{4}$ and influenza virus. ${ }^{5}$ Moreover, anti-MOG antibodies have been discovered in patients with $\mathrm{ADEM}^{5}$ or longitudinally extensive transverse myelitis ${ }^{4}$ following viral infection. Notably, the detection of anti-MOG antibodies is currently assumed to be related with NMSDS, rather than MS. ${ }^{38}$ For patients with a history of septicaemia, detection of anti-MOG and aquaporin-4 antibodies in serum is suggested, in the presence of a suspicion of demyelinating diseases, such as NMO, NMOSD and anti-MOG-associated spectrum. Although it has been proposed that acute viral infection could trigger autoimmunity via molecular mimicry mechanism, it remains unclear whether infection could trigger the NMO-associated autoimmunity. ${ }^{39}$ In spite of the extensive studies on NMO, NMOSD and anti-MOG-associated spectrum following infections, patients without a history of septicaemia can also develop demyelinating diseases due to other autoimmunity disorders. In summary, septicaemia could cause damages to oligodendrocytes and accordingly increase the risk of developing demyelinating diseases through various mechanisms.

\section{CONCLUSIONS}

Our results showed that the presence of septicaemia was prominently correlated with developing subsequent NMSDS. The increased risk of NMSDS is greater in septicaemia patients aged below 65 years and those with more hospitalisation days. Notably, the findings of this study point out the importance and necessity of thorough management strategies for septicaemia. Further studies are needed to verify whether the early intervention for septicaemia can reduce the risk of acquiring NMSDS. 


\section{LIMITATIONS}

There are several limitations in this study. First, we could not access the detailed information about life styles of the patients with NMSDS, including intensity of tobacco use, severity of functional disability, laboratory data and image studies because NHI Research Database originating from an administrative coding data set undersupplied above details. Second, we could not evaluate the severity in detail and the prognosis of demyelinating diseases after septicaemia. A prospective and longitudinal study will be required to investigate the association between cytokine change during septicaemia and the severity of NMSDS. Third, the participants were recruited according to ICD-9 codes, and this could give rise to potential misclassification bias in our analyses. A cross-checking system monitoring the correctness of records from diverse hospitals is therefore being intensively conducted by the NHI administration in Taiwan. Furthermore, the diagnosis of several demyelinating diseases such as ADEM can only be made by longitudinally following patients to ensure they do not develop any further CNS demyelinating lesions which would then fulfil the criteria of MS. In fact, the average annual incidence rate of MS in Taiwan was $0.79 / 100000$ around, lower than many high-latitude areas, ${ }^{40}$ and we therefore focused on the NMSDS in the present study.

\section{Main messages}

Septicaemia is positively correlated with subsequent nonmultiple sclerosis demyelinating syndromes (NMSDS).

- The increased risk of NMSDS is greater in septicaemia patients aged below 65 years and those with more hospitalisation days.

- This study points out the importance of thorough management strategies for septicaemia, which possibly contributes to increased hazard of NMSDS.

\section{Current research questions}

- Could interventions for septicaemia have beneficial effects on onset of non-multiple sclerosis demyelinating syndromes (NMSDS)?

- What inflammatory mediator(s) responding to septicaemia is correlated to development of NMSDS?

\section{Author affiliations}

${ }^{1}$ Department of Neurology, Tri-Service General Hospital, National Defense Medical Center, Taipei, Taiwan, Republicof China

${ }^{2}$ Graduate Institute of Medical Sciences, National Defense Medical Center, Taipei, Taiwan, Republic of China

${ }^{3}$ Department of Neurology, Shin-Kong WHS Memorial Hospital, Taipei, Taiwan, Republic of China

${ }^{4}$ School of Medicine, College of Medicine, Taipei Medical University, Taipei, Taiwan, Republic of China

${ }^{5}$ Division of Neurology, Department of Medicine, Cheng Hsin General Hospital, Taipei, Taiwan, Republic of China

${ }^{6}$ Management Office for Health Data, China Medical University Hospital, College of Medicine, China Medical University, Taichung, Taiwan, Republic of China

${ }^{7}$ Graduate Institute of Clinical Medical Science, China Medical University, Taichung, Taiwan, Republic of China

${ }^{8}$ Department of Internal Medicine, College of Medicine, China Medical University, Taichung, Taiwan, Republic of China

${ }^{9}$ Division of Kidney Disease, China Medical University Hospital, Taichung, Taiwan, Republic of China

${ }^{10}$ Department of Neurology, China Medical University Hospital, Taichung, Taiwan, Republic of China

Contributors Study concept/design: CHC, CKT and JTL; data collection and analysis: CHC, CKT, JTL, IJT, IKW, CHT and CYH; data interpretation: CHC, CKT, LML, JTL, JHY,
CCL, YFS, FCY and CLT; manuscript writing: CHC, CKT and JTL. All authors have given their final approval of the version to be published.

Funding This work was supported in part by grants from the Ministry of Science and Technology (MOST 106-2314-B-016-010, MOST 105-2314-B016-004 and MOST 106-2314-B-016-007-MY2), Ministry of National Defense Medical Affairs Bureau (MAB-106-041 and MAB-106-044), Tri-Service General Hospital (TSGH-C105-082, TSGH-C107-073, TSGH-C 106-068, TSGH-C107-072, TSGH-C107-074 and TSGH-C108-006-007-007-S05), Cheng Hsin General Hospital (CH-NDMC-106-13), the Ministry of Health and Welfare, Taiwan (MOHW107TDU-B-212-123004), China Medical University Hospital, Academia Sinica Taiwan Biobank Stroke Biosignature Project (BM10701010021), Taiwan Clinical Trial Consortium for Stroke (MOST 106-2321-B-039-005), Tseng-Lien Lin Foundation, Taichung, Taiwan, Taiwan Brain Disease Foundation, Taipei, Taiwan, and Katsuzo and Kiyo Aoshima Memorial Funds, Japan.

Competing interests No, there are no competing interests for any author. Patient consent for publication Not required.

Provenance and peer review Not commissioned; externally peer reviewed.

Data sharing statement Data are available in a public, open access repository. There are no data in this work. Data are available upon reasonable request. Data may be obtained from a third party and are not publicly available. No data are available. All data relevant to the study are included in the article or uploaded as supplementary information.

Open access This is an open access article distributed in accordance with the Creative Commons Attribution Non Commercial (CC BY-NC 4.0) license, which permits others to distribute, remix, adapt, build upon this work non-commercially, and license their derivative works on different terms, provided the original work is properly cited, appropriate credit is given, any changes made indicated, and the use is non-commercial. See: http://creativecommons.org/licenses/by-nc/4.0/.

\section{REFERENCES}

1 Bradl M, Lassmann H. Oligodendrocytes: biology and pathology. Acta Neuropathol 2010;119:37-53.

2 Karussis D, Petrou P. The spectrum of post-vaccination inflammatory CNS demyelinating syndromes. Autoimmun Rev 2014;13:215-24.

3 Singer M, Deutschman CS, Seymour CW, et al. The third International consensus definitions for sepsis and septic shock (Sepsis-3). JAMA 2016;315:801-10.

4 Nakamura Y, Nakajima $\mathrm{H}$, Tani $\mathrm{H}$, et al. Anti-MOG antibody-positive ADEM following infectious mononucleosis due to a primary EBV infection: a case report. BMC Neurol 2017;17.

5 Amano H, Miyamoto N, Shimura H, et al. Influenza-associated MOG antibody-positive longitudinally extensive transverse myelitis: a case report. BMC Neurol 2014;14.

6 Rees J. Complex disease and the new clinical sciences. Science 2002;296:698-700.

7 Lincoln MR, Montpetit A, Cader MZ, et al. A predominant role for the HLA class II region in the association of the $\mathrm{MHC}$ region with multiple sclerosis. Nat Genet 2005;37:1108-12.

8 Pierrot-Deseilligny C, Souberbielle J-C. Is hypovitaminosis D one of the environmental risk factors for multiple sclerosis? Brain 2010;133:1869-88.

9 Mikaeloff Y, Caridade G, Tardieu M, et al. Parental smoking at home and the risk of childhood-onset multiple sclerosis in children. Brain 2007;130:2589-95.

10 Briggs FBS, Acuña BS, Shen L, et al. Adverse socioeconomic position during the life course is associated with multiple sclerosis. J Epidemiol Community Health 2014;68:622-9.

11 Eduwu J, Tabasam F, Bastidas AA, et al. Successful management of methicillinresistant Staphylococcus aureus bacteremia complicated with diffuse myelitis. Infect Dis 2017:49:234-6.

12 Richert ME, Hosier $\mathrm{H}$, Weltz AS, et al. Acute transverse myelitis associated with Salmonella Bacteremia: a case report. Am J Case Rep 2016;17:929-33.

13 Semmler A, Hermann S, Mormann F, et al. Sepsis causes neuroinflammation and concomitant decrease of cerebral metabolism. J Neuroinflammation 2008;5.

14 Barichello T, Fortunato JJ, Vitali AM, et al. Oxidative variables in the rat brain after sepsis induced by cecal ligation and perforation. Crit Care Med 2006;34:886-9.

15 Taccone FS, Su F, Pierrakos C, et al. Cerebral microcirculation is impaired during sepsis: an experimental study. Crit Care 2010;14.

16 Iwashyna TJ, Ely EW, Smith DM, et al. Long-term cognitive impairment and functional disability among survivors of severe sepsis. JAMA 2010;304:1787-94.

17 Tate JA, Snitz BE, Alvarez KA, et al. Infection hospitalization increases risk of dementia in the elderly. Crit Care Med 2014;42:1037-46.

18 Lee J-T, Chung WT, Lin J-D, et al. Increased risk of stroke after septicaemia: a population-based longitudinal study in Taiwan. PLoS One 2014;9:e89386.

19 Watad A, Bragazzi NL, Adawi M, et al. Autoimmunity in the Elderly: Insights from Basic Science and Clinics - A Mini-Review. Gerontology 2017;63:515-23.

20 Vincent A, Lahr BD, Wolfe F, et al. Prevalence of fibromyalgia: a population-based study in Olmsted County, Minnesota, utilizing the Rochester epidemiology project. Arthritis Care Res 2013;65:786-92. 
21 Pompa A, Clemenzi A, Troisi E, et al. Chronic pain in multiple sclerosis patients: utility of sensory quantitative testing in patients with fibromyalgia comorbidity. Eur Neurol 2015;73:257-63.

22 Mawanda F, Wallace RB, McCoy K, et al. Systemic and localized extra-central nervous system bacterial infections and the risk of dementia among US veterans: a retrospective cohort study. Alzheimers Dement 2016;4:109-17.

23 Lindsberg PJ, Grau AJ. Inflammation and infections as risk factors for ischemic stroke. Stroke 2003;34:2518-32.

24 Serlin Y, Shelef I, Knyazer B, et al. Anatomy and physiology of the blood-brain barrier. Semin Cell Dev Biol 2015;38:2-6.

25 Handa O, Stephen J, Cepinskas G. Role of endothelial nitric oxide synthase-derived nitric oxide in activation and dysfunction of cerebrovascular endothelial cells during early onsets of sepsis. Am J Physiol Heart Circ Physiol 2008;295:H171 2-H1719.

26 Terborg C, Schummer W, Albrecht M, et al. Dysfunction of vasomotor reactivity in severe sepsis and septic shock. Intensive Care Med 2001;27:1231-4.

27 Taccone FS, Castanares-Zapatero D, Peres-Bota D, et al. Cerebral autoregulation is influenced by carbon dioxide levels in patients with septic shock. Neurocrit Care 2010;12:35-42.

28 Yao SY, Ljunggren-Rose A, Chandramohan N, et al. In vitro and in vivo induction and activation of nNOS by LPS in oligodendrocytes. J Neuroimmunol 2010;229:146-56.

29 Faix JD. Biomarkers of sepsis. Crit Rev Clin Lab Sci 2013;50:23-36.

30 Holub M, Lawrence DA, Andersen N, et al. Cytokines and chemokines as biomarkers of community-acquired bacterial infection. Mediators Inflamm 2013;2013:1-7.
31 Hannestad J, Gallezot J-D, Schafbauer T, et al. Endotoxin-induced systemic inflammation activates microglia: $\left[{ }^{11} \mathrm{C}\right]$ PBR28 positron emission tomography in nonhuman primates. Neuroimage 2012;63:232-9.

32 Moreno B, Jukes J-P, Vergara-Irigaray N, et al. Systemic inflammation induces axon injury during brain inflammation. Ann Neurol 2011;70:932-42.

33 Horwitz MS, Evans CF, Mcgavern DB, et al. Primary demyelination in transgenic mice expressing interferon-gamma. Nat Med 1997;3:1037-41.

34 Campbell IL. Structural and functional impact of the transgenic expression of cytokines in the CNS. Ann N Y Acad Sci 1998;840:83-96.

35 Davies DC. Blood-brain barrier breakdown in septic encephalopathy and brain tumours. J Anat 2002;200:639-46.

36 Merkler $D$, Horvath $E$, Bruck W, et al. "Viral déjà vu" elicits organ-specific immune disease independent of reactivity to self. J Clin Invest 2006;116:1254-63.

37 Evans CF, Horwitz MS, Hobbs MV, et al. Viral infection of transgenic mice expressing a viral protein in oligodendrocytes leads to chronic central nervous system autoimmune disease. J Exp Med 1996;184:2371-84.

38 Ramanathan S, Dale RC, Brilot F. Anti-MOG antibody: the history, clinical phenotype, and pathogenicity of a serum biomarker for demyelination. Autoimmun Rev 2016:15:307-24

39 Jasiak-Zatonska M, Kalinowska-Lyszczarz A, Michalak S, et al. The immunology of neuromyelitis Optica-Current knowledge, clinical implications, controversies and future perspectives. Int J Mol Sci 2016;17.

40 Lai C-H, Tseng H-F. Population-based epidemiological study of neurological diseases in Taiwan: I. Creutzfeldt-Jakob disease and multiple sclerosis. Neuroepidemiology 2009;33:247-53 